Vol. 13, $n^{\circ} 1 \mid 2009$

Varia

\title{
L'observation des relégués en France (1947-1970)
}

Jean-Claude Vimont

\section{OpenEdition}

Journals

Édition électronique

URL : https://journals.openedition.org/chs/691

DOI : $10.4000 /$ chs. 691

ISSN : 1663-4837

Éditeur

Librairie Droz

Édition imprimée

Date de publication : 1 mars 2009

Pagination : 49-72

ISBN : 978-2-600-01295-9

ISSN : 1422-0857

Référence électronique

Jean-Claude Vimont, "L'observation des relégués en France (1947-1970) », Crime, Histoire \& Sociétés / Crime, History \& Societies [En ligne], Vol. 13, n¹ | 2009, mis en ligne le 01 mars 2012, consulté le 23 mars 2022. URL : http://journals.openedition.org/chs/691 ; DOI : https://doi.org/10.4000/chs.691 


\title{
L'observation des relégués en France (1947-1970)
}

\author{
Jean-Claude Vimont ${ }^{1}$
}

\begin{abstract}
The individualization of sentences was at the heart of the Prison Reform in France after 1945 to identify the personality of a convict called for increased instances of observation and cross social surveys with psychological, psychiatric and medical expertise. From 1948 to 1970, repeated offenders sentenced to an additional sentence of security have been the object of an experiment on a large scale. The analysis of their prison records, of their personality records show the gap between the generous intentions of the promoters of these observations and the practices of field observers. Many aspects of an old-fasioned form of criminology influence the whiters of the reports along with many prejudices. They do little to shorten the journey to freedom, but actuelly lengthen the duration of the sentence.
\end{abstract}

L'individualisation des peines fut au cour de la Réforme pénitentiaire en France après 1945. Cerner la personnalité d'un condamné exigeait de multiplier les instances d'observation et de croiser les enquêtes sociales avec des expertises psychologiques, psychiatriques et médicales. Les récidivistes condamnés à une peine complémentaire de sûreté ont fait l'objet, de 1948 à 1970, d'une expérimentation à grande échelle. L'analyse de leurs dossiers pénitentiaires, de leurs dossiers de personnalité montre l'écart entre les intentions généreuses des promoteurs de ces observations et les pratiques des observateurs du terrain. Bien des aspects d'une criminologie éculée influencent les rédacteurs des rapports aux côtés de nombreux préjugés. Ils n'aident guère à abréger le cheminement vers la liberté, mais allongent la durée de la peine.

A près la Seconde Guerre mondiale, l'Administration pénitentiaire française mène une expérimentation à grande échelle en direction des condamnés à la peine complémentaire de la relégation qui, de 1885 à 1970, sanctionna les délinquants multirécidivistes. La clé de voûte de cette entreprise est l'observation, l'examen médico-psychologique et social des condamnés afin d'individualiser les sanctions et de déterminer un cheminement carcéral adapté à leur personnalité. Cette

\footnotetext{
L'auteur est maître de conférences d'histoire contemporaine et Directeur du département d'histoire de l'Université de Rouen. Il a publié notamment: La Prison politique en France, genèse d'un mode d'incarcération spécifique, XVIII ${ }^{e}-X X^{e}$ siècles, Paris, Anthropos, 1993; La prison, à l'ombre des hauts murs, Paris, Gallimard, 2004; Jeunes, déviances et identités, XVIII ${ }^{e} X X^{e}$ siècles, Rouen, PURH, 2005. Ses recherches actuelles portent sur les relégués et la Réforme pénitentiaire après 1945.
} 
expérience s'inspire des principes de politique criminelle théorisés par Marc Ancel, la Défense sociale nouvelle, et est l'une des applications de la Réforme pénitentiaire initiée dès la Libération par Paul Amor. La «nouvelle » relégation espère mener progressivement le plus grand nombre de ceux qui en sont atteints vers la liberté et la réinsertion sur le territoire métropolitain de la République et donc rompre avec la nature éliminatoire de cette peine complémentaire de sûreté qui était antérieurement appliquée dans les territoires d'outre-mer. L'analyse de plus d'un millier de dossiers de relégués ${ }^{2}$ montre les limites de cette innovation pénale. L'écart est flagrant entre les principes affichés par ses promoteurs et les pratiques des personnels ayant en charge ces détenus. Persiste l'une des finalités de cette peine complémentaire, de cette mesure de sûreté, la mise à l'écart pendant de très longues années de condamnés plus malchanceux que dangereux.

La peine complémentaire de la relégation a été promulguée le 27 mai 1885. Votée à l'initiative de gouvernements républicains «opportunistes», soucieux de satisfaire une opinion irritée par la persistance de la mendicité, du vagabondage, de la petite délinquance d'habitude dans un contexte de crise économique et désireux de montrer à une clientèle électorale d'artisans, de commerçants et de paysans que le régime républicain était capable d'assurer l'ordre en même temps que le progrès, cette loi d'une sévérité extrême organise l'éloignement en Guyane et en NouvelleCalédonie de près de 30000 récidivistes de 1885 à 1938 , date du dernier convoi vers Saint-Jean-du-Maroni en Guyane. Albert Camus, en 1938, rédige dans Alger Républicain un article titré «Ces hommes qu'on raye de l'humanité» pour décrire cet ultime transport et évoquer cette peine d'élimination, de débarras. La relégation est une peine complémentaire et perpétuelle de droit commun subie à l'expiration de la peine principale. Elle est automatiquement prononcée par le juge de la dernière sentence au vu des condamnations antérieures subies par l'accusé. Si, dans un intervalle de dix années, les condamnations antérieures et l'ultime condamnation aboutissent aux quatre ensembles suivants, la relégation est appliquée: deux condamnations criminelles aux travaux forcés ou à la réclusion; une condamnation aux travaux forcés ou une condamnation à la réclusion et trois condamnations à plus de trois mois d'emprisonnement; quatre condamnations à plus de trois mois d'emprisonnement; sept condamnations dont deux à plus de trois mois d'emprisonnement et cinq pour vagabondage ou infraction à l'interdiction de résidence à condition que deux des cinq dernières aient été de plus de trois mois d'emprisonnement. La relégation est appliquée aux hommes et aux femmes jusqu'en 1907, aux hommes seulement ensuite. Une commission de classement auprès du ministre de l'Intérieur, puis à partir de 1912 auprès du ministre de la Justice, décide du mode d'effectuation de la relégation. Pour une infime minorité de relégués pourvus de moyens d'existence ou d'un métier, il ne s'agit que d'un exil dans la colonie désignée. C'est la relégation individuelle. Pour la très grande majorité, la peine s'effectue dans un établissement pénitentiaire avec astreinte au travail. C'est la relégation collective. Dans ce second cas, leur sort est comparable à celui des condamnés aux travaux forcés. Cette loi sécuritaire rencontre très vite l'hostilité des magistrats qui en restreignent l'applica-

2 Dans le cadre d'une recherche en cours sur les relégués et la réforme pénitentiaire, nous avons pu consulter l'ensemble des dossiers du Centre de triage et d'observation de Rouen, ceux du Centre de relèvement par le travail de l'Armée du Salut à Radepont dans l'Eure, une sélection de dossiers du Centre de semi-liberté de Clermont-Ferrand et les dossiers de la prison d'antisociaux de Gannat dans l'Allier. 
tion. En prononçant des sentences inférieures à trois mois et un jour de prison à l'encontre des mendiants, vagabonds et petits voleurs, ils évitent une peine perpétuelle qui heurte le principe de la proportionnalité de la peine au délit. L'automaticité de la relégation contredit l'individualisation des sentences pénales. Les rigueurs du séjour en Guyane, le taux de mortalité des transportés, la misère et la corruption du camp de Saint-Jean-du-Maroni, l'échec de toute colonisation pénale en font une sentence excessive que dénoncent tous les visiteurs de la Guyane pendant les années trente. Les journalistes Albert Londres et Alexis Danan, le major Péan de l'Armée du Salut, des médecins et des administrateurs pointent l'échec de la relégation lors de la campagne d'opinion qui met en accusation les bagnes coloniaux.

Dès 1937, une commission ministérielle prépare la transformation des peines coloniales en peines métropolitaines et envisage un nouveau mode de relégation. Le décret-loi du 17 juin 1938 qui supprime les travaux forcés outre-mer laisse de côté la relégation. Il faut attendre l'acte dit loi du 6 juillet 1942, qui reprend les propositions de la commission de 1937, pour que naisse la relégation métropolitaine. L'horizon a changé. Les relégués subissent la peine complémentaire dans un établissement ou un quartier de prison spécialement aménagé à leur destination. Ils demeurent soumis à l'obligation de travail et leur conduite journalière doit être évaluée afin de mesurer leurs efforts de réadaptation. À l'issue de trois années, ils peuvent être libérés conditionnellement par arrêté du garde des Sceaux. Cette loi n'est pas abolie aux lendemains de la Libération et demeure en vigueur jusqu'en 1954. À cette date, à l'initiative du député Jacques Isorni, le parlement vote la fin de l'automaticité de la relégation, les juges retrouvant la faculté de la prononcer ou de ne pas le faire. Le nombre de condamnations à la relégation diminue sensiblement et des voix de plus en plus nombreuses s'élèvent contre cette sentence perpétuelle archaïque et stigmatisante. Elle est finalement supprimée en 1970, remplacée jusqu'en 1981 par la tutelle pénale au champ d'application beaucoup plus restreint et qui ne concerne que quelques dizaines de condamnés.

Organiser la relégation en métropole est l'une des tâches prioritaires assignée aux magistrats qui reprennent en main l'administration pénitentiaire après les dérives fascistes de ses chefs pendant le premier semestre de 1944. La tâche n'est pas mince, car le parc carcéral est en piteux état, détérioré, misérable, régi par une discipline de fer, encombré des milliers de justiciables des Cours de justice, prévenus puis condamnés pour faits de collaboration, des condamnés aux travaux forcés et des relégués qui ont connu la faim, survécu aux maladies et à la hausse de la mortalité des années de guerre. Paul Amor est nommé directeur de l'administration pénitentiaire. Dès 1944, une commission est mise en place auprès du ministre de la Justice pour élaborer une réforme pénitentiaire. Son secrétaire est Pierre Cannat. Un programme de quatorze points fixe les grandes orientations. Le quatorzième concerne les relégués. Ils doivent être soumis «à un internement en colonie pénale, en principe perpétuel, mais susceptible d'être abrégé par une libération anticipée». Un réseau d'établissements spécifiques est mis en place en métropole: camps pénitentiaires, centres de triage et d'observation, prisons et quartiers de maisons centrales, centres de semi-liberté et foyers de réinsertion. Les relégués ${ }^{3}$ y séjournent

Ils sont 623 au $1^{\text {er }}$ janvier 1946, 931 au $1^{\text {er }}$ janvier 1950, 1118 au $1^{\text {er }}$ janvier 1951, 1375 au $1^{\text {er }}$ janvier 1952, 1536 au $1^{\text {er }}$ janvier 1953, 1647 au $1^{\text {er }}$ janvier 1954 (soit près de 8\% d'une population totale de 22662 détenus). La loi du 3 juillet 1954 rend l'application de la peine complémentaire de la relégation facultative. Ils sont 1635 (8,6\% des 18992 détenus) au $1^{\text {er }}$ janvier 1955, 1548 (8,2\% 
plus ou moins longuement et cheminent progressivement vers la libération conditionnelle envisagée par la loi du 6 juillet 1942, au terme d'un délai d'épreuve minimum de trois années. Le parcours est semé d'embûches, car la liberté n'est pas accordée automatiquement. Le comportement des relégués est sans cesse scruté, noté, évalué. Leur passé pénal, leurs antécédents familiaux, leur personnalité, leur attitude et leur physique sont analysés. Ils comparaissent devant des commissions de classement ou de mise en liberté qui peuvent repousser la date de la mise en liberté et les transférer dans un établissement adapté à leur personnalité.

Les initiateurs du dispositif, au premier rang desquels Pierre Cannat, directeur adjoint de l'Administration pénitentiaire, véritable cheville ouvrière de cette réforme, souhaitent prémunir la société de tout risque car ils n'ont qu'une confiance très limitée dans la capacité de réinsertion d'une large fraction de ces récidivistes, ceux qu'ils qualifient d'asociaux, et se défient d'une minorité de réfractaires à la discipline pénitentiaire qu'ils érigent en une catégorie, les antisociaux. Certes, Pierre Cannat, dans son livre Nos frères les récidivistes ${ }^{4}$, avait affirmé sa croyance dans l'amendement de tout homme et s'était élevé contre la mesure d'élimination et de débarras qu'avait été la relégation de 1885 à 1938. En 1950, de retour d'un voyage d'études aux États-Unis, il avait vanté les nouvelles tendances d'un droit pénal «baigné de charité et d'humanité » ${ }^{5}$. Mais, partisan des mesures de sûreté à durée indéterminée ${ }^{6}$ et influencé par les courants criminologiques qui préconisaient de soumettre tout délinquant à un examen médico-psychologique et social, il balise ce cheminement vers la liberté de nombreux gardes-fous.

Pierre Cannat s'inspirait de la Défense sociale nouvelle théorisée par Marc Ancel $^{7}$. Ce magistrat, détaché au ministère de la Justice en 1936, était depuis cette année-là le secrétaire général de l'Institut de Droit Comparé et le rédacteur en chef de la Revue de Science criminelle et de droit comparé, principal vecteur de sa théorie. Il affirmait le droit pour toute société de se prémunir de la dangerosité de certains individus, récidivistes, délinquants «anormaux mentaux » dont la personnalité, qu'il convenait d'analyser, manifestait des signes d'asocialité décelables quelle que soit la gravité du délit commis. Mais il substituait aux mesures d'élimination, de ségrégation préconisées pendant les premières décennies du siècle, un objectif de resocialisation du déviant. Par stades progressifs, les pénitentiaires et les juges, les éducateurs et les assistantes sociales, ces nouveaux acteurs de la réforme pénitentiaire, devaient, en lui reconnaissant sa dignité d'homme et en lui faisant confiance, accompagner celui qui était frappé d'une mesure de sûreté vers la liberté. Marc Ancel, devenu après-guerre Conseiller à la Cour de Paris, ne cessa de défendre cette

des 18780 détenus) au $1^{\text {er }}$ janvier 1956, 1447 au $1^{\text {er }}$ janvier 1957, 1730 (sur 23360 détenus) au $1^{\text {er }}$ janvier 1958, à la suite du rapatriement en métropole de relégués d'Afrique du Nord (environ 400 placés dans la maison centrale d'Eysses), 1552 (5,6\% de 28386 détenus) au $1^{\text {er }}$ janvier 1959, 1419 au $1^{\text {er }}$ janvier 1960,1310 au $1^{\text {er }}$ janvier 1961, 1235 au $1^{\text {er }}$ janvier 1962.

4 Cannat, 1942.

5 «L'homme devient le centre du droit pénal. Il est de plus en plus jugé sur sa valeur globale et non plus seulement sur sa faute d'un jour; la peine qui lui est imposée n'est plus un carcan de misère, mais un moyen de l'aider à devenir meilleur. En somme dans ce domaine, l'horizon est clair», Cannat (1950, p. 170).

6 Sur la question centrale des mesures de sûreté et des sentences indéterminées, voir, entre autres Ancel (1950).

7 Ancel (1954). 
politique criminelle humaniste mais souligna la difficulté de l'entreprise en direction des multirécidivistes relégués. Il insistait sur les efforts de classification:

Les mesures de sûreté à appliquer aux récidivistes restent sans aucun doute le point le plus délicat. La France et la Belgique ont récemment fait sur ce point des expériences qui, jusqu'à présent, n'ont pas été entièrement couronnées de succès. Pour certains délinquants d'habitude, le problème de la rééducation présente des difficultés considérables. Aussi les différents systèmes pénitentiaires ont-ils un peu tendance, pour certains de ces délinquants, à en revenir à un régime qui se rapproche, au point de se confondre souvent, avec celui de l'emprisonnement ou de la réclusion. Néanmoins, il serait exagéré de soutenir que, pour la catégorie des relégués (au sens français du mot) par exemple, tout effort de reclassement doive être abandonné. Sans doute est-ce par un effort nouveau de classification et de distinction des condamnés en groupes différents que la solution du problème sera recherchée dans les années à venir .

L'observation est donc au centre du processus. Elle est menée par les pénitentiaires des différents établissements, par les magistrats qui président les commissions de libération conditionnelle et qui dirigent les comités d'assistance aux libérés, puis à partir de 1958 par les juges d'application des peines, par des psychologues, des psychotechniciens, des psychiatres, des assistantes sociales et des éducateurs. Ils ont à leur disposition les outils d'une criminologie assez disparate que Jean Pinatel ${ }^{9}$ vulgarise dans ses chroniques régulières de la Revue de science criminelle et de droit pénal comparé.

Des sessions d'observation que nous qualifierions aujourd'hui de pluridisciplinaires sont organisées dans les centres d'observation et de triage mis en place à partir de 1947 à Loos-lès-Lille d'abord, puis à Rouen ouvert en 1952, à Besançon en août 1953 et à Saint-Étienne en septembre 1955. Elles s'appuient sur d'autres observations pratiquées lors des expertises psychiatriques réclamées pendant une instruction, par les pénitentiaires lorsque le détenu a séjourné dans une prison à régime progressif ou lorsqu'il a effectué un stage au Centre national d'orientation de Fresnes, par les assistants sociaux et les éducateurs des différents centres de relégués. Elles s'accumulent au sein de véritables «casiers pénitentiaires» qui suivent les relégués tout au long de leurs pérégrinations dans l'univers carcéral spécifiquement aménagé à leur intention. Typologies et catégories apparaissent alors, «délinquants ethniques», «délinquants constitutionnels», «ivrognes d'habitude», «relégués-types », qui pèsent considérablement sur le devenir d'hommes qui demeurent incarcérés bien au delà du délai théorique des trois années, envisagé en 1942.

Le corps des relégués est l'objet d'une attention toute particulière de la part de ces nombreux observateurs, car ils pensent y repérer les stigmates de maladies mentales, de pathologies héréditaires, de dysfonctionnements ou dysmorphismes dits constitutionnels. Les données anthropométriques et morphologiques complètent des dossiers médicaux qui ne négligent ni la robustesse, le tempérament, le biotype, l'usure organique, les infirmités, les handicaps et les lésions. Les longs séjours en prison usent les corps, vieillissent prématurément les êtres. La tuberculose et bien d'autres handicaps frappent cette population particulière. L'alcool est également au centre des préoccupations.

Ancel (1950b, pp. 48-49).

Pinatel (1960). 
Pour évoquer les processus d'observation dans l'orientation des relégués, nous nous appuyons sur une analyse de 1465 dossiers: 740 provenant du centre d'observation de Rouen ${ }^{10}, 266$ provenant du centre de semi-liberté de Clermont-Ferrand ${ }^{11}$, 459 provenant du centre salutiste de Radepont dans l'Eure ${ }^{12}$, bien souvent la dernière étape pour ceux qui ne parviennent que difficilement à retrouver le chemin de la liberté.

\section{UN CHEMIN DE CROIX PARSEMÉ D'INSTANCES D'OBSERVATION}

Avant d'entamer la peine complémentaire de relégation, le détenu subit la peine de prison qui l'a entraînée. Si elle est courte, ce qui est le plus souvent le cas puisque les relégués sont majoritairement des voleurs de petite envergure, elle est accomplie dans une maison d'arrêt et de correction. S'il s'agit d'une peine de réclusion ou de travaux forcés, peines infligées par les cours d'assises aux auteurs de vols avec circonstances aggravantes, elle conduit le condamné dans une maison centrale où des notices et appréciations vont accompagner son séjour: celles du Centre national d'orientation de Fresnes qui a décidé de l'affectation, celles du «Bulletin de statistique morale» tenu dans les maisons centrales ordinaires et enrichi des rapports d'incidents, celles des instances d'observation dans les maisons centrales réformées à régime progressif qui décident du passage d'une classe dans une autre.

Âl'issue de cet emprisonnement, qui succède à bien d'autres, le « relégué peine accomplie» est transféré dans les centres pénitentiaires d'épreuve à Saint-Martinde-Ré ou à Mauzac. La maison centrale de Fontevraud accueillit quelques temps des relégués lorsque cette population atteint des sommets en 1954. Mais des incidents relatés par René Boloux, auteur de l'un des rares témoignages sur la relégation après-guerre, mirent fin à cette solution de désencombrement. Astreint au travail forcé pendant trois ans, le relégué fait l'objet d'une fiche de notation de la part du directeur. De son côté, il prépare un dossier de libération conditionnelle et s'efforce de réunir certificats de travail et d'hébergement pour la commission qui va statuer à l'issue des trois années dites d'épreuve. Les assistants sociaux des centres, membres de l'Armée du Salut à Saint-Martin-de-Ré, les conseillent et les aident. Ils recommandent certains pour des institutions de l'œuvre, comme le foyer de relèvement par le travail, dans le château de Radepont, dans l'Eure. La commission présidée par un magistrat décide la libération conditionnelle directe qui permet de désencombrer les centres d'épreuve au milieu des années cinquante, la libération conditionnelle avec obligation d'un séjour probatoire d'une année chez les salutistes de Radepont, le transfert dans un centre de triage pour un stage d'observation.

Quatre centres de triage accueillent pour six mois des convois de relégués: Lille, Rouen, Saint-Étienne et Besançon. Ils y sont soumis à un régime progressif accé-

10 J'ai organisé en mars 2005 la récupération dans les greniers de la prison Bonne Nouvelle de Rouen des dossiers du centre de triage qui fonctionna de 1952 à 1970. Ils figurent désormais dans 87 cartons.

11 Nous avons procédé à une sélection alphabétique au centre de semi-liberté de Clermont-Ferrand: dossiers de $\mathrm{A}$ à $\mathrm{C}$ et dossiers de $\mathrm{L}$ à W.

12 Le centre conserve des dossiers et des fichiers. Ils ont tous été analysés de 1953 à 1970. 
léré: trois mois d'isolement cellulaire avec quelques sorties promenades, trois mois de semi-liberté avec obligation de rentrer le soir dans la prison. Ils y sont observés par une équipe composée d'un éducateur, d'un directeur chef de centre, d'une assistante sociale, d'un psychiatre et d'un magistrat qui préside une commission de classement et d'orientation. Ils sont répartis alors en trois groupes: ceux qui sont admis et maintenus en libération conditionnelle (avec à Rouen l'aide du centre salutiste de Radepont), les asociaux destinés à des centres de semi-liberté, les antisociaux promis à un emprisonnement. Les nombreuses évasions lors des sorties-promenades, lors de la semi-liberté et dans les premiers temps de la libération conditionnelle grossissent les deux dernières catégories.

Les asociaux sont conduits au centre de semi-liberté Pélissier de ClermontFerrand, ouvert en avril 1950. Il portera ultérieurement le nom du docteur Giscard, médecin psychiatre qui présida ses premiers pas. Ils séjournent trois à quatre mois au centre, puis sont placés six mois en semi-liberté avant qu'une commission ne décide de leur admission à la liberté conditionnelle. Deux autres centres de semiliberté sont adjoints au premier, le centre de semi-liberté Pescayre à Saint-Sulpice, dans le Tarn, ouvert en août 1952 pour les asociaux ruraux qui peuvent trouver un emploi dans les fermes environnantes, et le centre de semi-liberté Boudet à Bordeaux, ouvert en octobre 1957 lorsque l'effectif total des relégués dépassait les 1500. Les centres de semi-liberté reçoivent également les antisociaux qui ont été incarcérés plusieurs années dans les prisons de Gannat et de Lure. Évasions et incidents émaillent les séjours dans ces centres. Les relégués repris après un délit sont conduits pour un stage disciplinaire dans les maisons centrales de Nîmes et de Riom. Ensuite, ils reprennent le circuit à son seuil, les centres d'épreuve de Ré et de Mauzac.

Les antisociaux sont incarcérés dans deux petites prisons, à Gannat, ouverte en mars 1951 et à Lure, ouverte en 1954. Sécurité maximum, mais régime débonnaire dans ces geôles à petit effectif. Une commission décide des transferts, après deux, trois ou quatre années, dans les centres de semi-liberté.

La libération conditionnelle pour une durée de vingt années suppose bien des contraintes et n'autorise guère d'écarts. Les échecs sont nombreux: récidives de vagabondage, d'infractions à l'interdiction de séjour qui entrave bien des entreprises de réinsertion, d'ivresse sur la voie publique et, bien sûr, récidive de menus larcins. Quelques relégués comprennent vite la logique du système et parviennent à disparaître à l'échéance des trois années d'épreuve. Pour beaucoup d'autres, le parcours est bien plus long, avec plusieurs essais en centre de triage, des allers et retours à Mauzac, à Clermont-Ferrand et même chez les officiers de l'Armée du Salut à Radepont. Il prolonge bien souvent un premier parcours carcéral dans les maisons centrales et même dans les camps allemands. Citons le parcours de «Pas de chance $»^{13}$, relégué antisocial tatoué jusqu'au front de cette formule qui ne facilitait guère son reclassement et qui s'évanouit du centre de Clermont-Ferrand en 1964.

Né en 1911, il subit une première condamnation de 1930 à 1932 dans la maison centrale de Clairvaux. Conséquence de cette délinquance initiale, il effectue son service militaire dans les Bat' d'Af' en Afrique du Nord de 1932 à 1936. Aux débuts de la guerre, il est de nouveau incarcéré, puis est conduit à Dachau de 1942 à 1945,

13 Archives départementales du Puy-de-Dôme, Dossier de Raymond B. 1124 W 256. 
parmi les triangles verts ${ }^{14}$. En 1948, il est condamné pour vol aggravé à 10 années de travaux forcés et à la relégation. Il est emprisonné dans la maison centrale de Poissy jusqu'en 1953. Il entame sa relégation à Saint-Martin-de-Ré en 1953 et y demeure jusqu'en 1957. Conduit au centre de triage de Rouen, il frappe un agent lors d'une sortie-promenade. Trois années supplémentaires à Saint-Martin-de-Ré. Il est conduit au centre de triage de Lille en 1961 et y est classé antisocial. Cela lui vaut trois années dans la prison de Gannat de 1962 à 1964. Il est ensuite conduit au centre Pélissier de Clermont-Ferrand. Il s'en évade peu de temps après y avoir été écroué.

Nous pourrions multiplier les itinéraires tragiques de relégués, sans cesse ramenés à la case prison pour de menus délits et des échecs en liberté. Les instances d'observation et de classement jalonnent ces parcours chaotiques et ne facilitent pas toujours la réinsertion prônée par les initiateurs de la réforme.

\section{DISTINGUER LES ANTISOCIAUX DES ASOCIAUX}

L'observation est étroitement associée au tri, au classement des délinquants afin de repérer ceux qui menacent réellement la société. Elle s'inspire des expériences menées en Belgique dès 1920 et en France à l'égard des mineurs dès les années trente $^{15}$. Pierre Cannat déplorait, en 1949, les mesures de sûreté indistinctement appliquées aux auteurs de délits semblables. Il citait l'exemple du vagabondage et préconisait d'étudier le passé des infracteurs afin de «frapper plus lourdement les innombrables malfaiteurs dont l'activité antisociale s'est manifestée avec modestie, mais qui portent en eux-mêmes les germes les plus dangereux de rébellion contre la loi commune ${ }^{16}$. Ce fut la mission assignée aux équipes d'observateurs et ceci dès l'ouverture du premier centre de tri dans le quartier cellulaire de la maison centrale de Loos-lès-Lille en avril 1948. Dès mars 1949, le directeur de l'Administration pénitentiaire Charles Germain, indiquait que les premiers mois de l'expérience révélaient qu'il y avait des catégories distinctes de relégués, des «antisociaux dangereux qu'on ne devra jamais rendre à la liberté», des «débiles divers dont l'aboulie paraît relever de l'asile ou du camp de travail » et quelques «rares sujets susceptibles, avec un bon encadrement, de retourner dans la vie libre $»^{17}$. Tous les rapports ultérieurs ${ }^{18}$ reprendront jusqu'en 1970 cette distinction entre les asociaux et les antisociaux ${ }^{19}$,

14 Les triangles verts marquaient les condamnés de droit commun, les triangles noirs les asociaux, selon les classifications nazies. Les dossiers d'observation ne comportent que rarement une vérification de la nature de la déportation des relégués. Les éducateurs se méfient des récits autobiographiques mentionnant des déportations pour raisons politiques.

15 Frank (1939).

16 Pierre Cannat, magistrat, contrôleur général des services pénitentiaires, 1949a, p. 43.

17 Charles Germain, directeur de l'Administration pénitentiaire, Rapport lu à la séance du Conseil supérieur de l'Administration pénitentiaire dans la séance du 29 mars 1949, Revue pénitentiaire et de droit pénal, 1949, pp. 133-134. Voir également le compte rendu de la réunion de la commission d'études de l'Administration pénitentiaire sur le problème des relégués du 10 mars 1949, Revue de Science criminelle et de droit pénal comparé, 1949, pp. 650-658.

18 Le premier bilan de l'expérience lilloise signé de Pierre Cannat, d'Albert Gayraud, directeur de la maison centrale de Lille, du psychiatre Vullien, du magistrat Vienne, est publié sous le titre «Le problème des relégués » dans la Revue pénitentiaire et de droit pénal, 1950, pp. 72-132.

19 Paul Amor, président de la section de Défense sociale de la commission sociale de l'ONU, utilise ces deux catégories à propos des mineurs de justice, Pella (1950, p. 137). 
avec les conséquences carcérales que nous venons de mentionner. Les enjeux de ces stages et pratiques d'observation ne sont donc pas négligeables et rencontrent l'approbation de tous les spécialistes des questions pénales réunis à La Haye en août 1950 lors du douzième congrès pénal et pénitentiaire international, congrès qui consacra l'une de ses séances au traitement des délinquants d'habitude et où s'illustrèrent Marc Ancel, Pierre Cannat et Albert Gayraud, directeur de la maison centrale de Lille. Jean Pinatel voyait dans les centres d'observation qui effectuaient un «pronostic social », qui sélectionnaient grâce à des méthodes médicales, psychologiques, pédagogiques et sociales, le lieu où la science pénitentiaire devenait une criminologie appliquée ${ }^{20}$.

En 1956, Roger Vienne, président du tribunal de Béthune en charge des libérations conditionnelles du centre de triage de Lille, fait une conférence sur la réadaptation des multirécidivistes. Il y dresse deux portraits des antisociaux et asociaux que nous pouvons présenter sous forme de tableau ${ }^{21}$ :

\section{Tableau 1. Les principaux traits des antisociaux et des asociaux selon Roger Vienne en 1956}

\begin{tabular}{|l|l|}
\hline Antisociaux & Asociaux \\
\hline Actifs & Défavorisés physiquement \\
\hline Intelligents & Niveau mental inférieur ou liminaire \\
\hline Forte volonté & Défaillance de la volonté \\
\hline $\begin{array}{l}\text { Agressivité plus ou moins avouée et qu'ils } \\
\text { savent dissimuler car soucieux de leur } \\
\text { intérêt }\end{array}$ & Instabilité et imprévoyance \\
\hline Cyniques & Souvent bâtards ou enfants abandonnés \\
\hline Mépris de la morale & Ouvriers agricoles ou manœuvres urbains \\
\hline Égoïstes & Suggestibles \\
\hline $\begin{array}{l}\text { Protestataires en prison, mais ils savent } \\
\text { dissimuler }\end{array}$ & $\begin{array}{l}\text { Acceptent toutes les disciplines, complexe } \\
\text { d'infériorité et de culpabilité }\end{array}$ \\
\hline $\begin{array}{l}\text { Ne se sont jamais livrés à un travail produc- } \\
\text { tif, horreur du travail régulier, infractions } \\
\text { graves préparées à l'avance avec des com- } \\
\text { plices et un savoir technique }\end{array}$ & $\begin{array}{l}\text { Chapardeurs, voleurs pour satisfaire les } \\
\text { besoins du moment }\end{array}$ \\
\hline $\begin{array}{l}\text { Avides de sensations primaires intenses, } \\
\text { dépensiers }\end{array}$ & Alcoolisme de compensation \\
\hline
\end{tabular}

20 Pinatel (1949, p. 721).

21 Compte rendu de la conférence de Roger Vienne, Revue pénitentiaire et de droit pénal, 1956, pp. 531-535. Texte de la conférence, «Considérations sur la psychologie, l'origine de l'état dangereux et les facteurs de réadaptation des multirécidivistes », Revue de Science criminelle et de droit pénal comparé, 1957, pp. 53-63. 
La notion d'antisocialité avait des contours flous. Le psychiatre Jean Dublineau ${ }^{22}$, à l'occasion du deuxième congrès international de Défense sociale, à Liège en octobre 1949, lui préférait celle de dyssocialité lorsqu'il s'agissait d'actes contraires aux intérêts du groupe, d'attitudes «en marge». Il restreignait l'antisocialité aux conduites hostiles au groupe ou à la loi. Lombroso et ses «criminels-nés », Dupré et ses «pervers instinctifs » semblaient avoir désigné des catégories d'antisociaux, mais leurs théories étaient largement remises en cause. Les études typologiques de personnalité proposaient un lien virtuel entre l'agressivité, le stade agressif et l'antisocialité. Mais Dublineau rappelait que les conduites sociales dépendaient étroitement du milieu. L'antisocialité était donc le versant social des états d'agressivité. Dans la pratique des observations, les antisociaux étaient les détenus dont les casiers judiciaires montraient qu'ils appartenaient au milieu, qu'ils étaient engagés dans la voie d'une délinquance professionnelle, qu'ils étaient des délinquants sexuels et tous ceux, la plus grande proportion de ce groupe, qui n'acceptaient pas la peine de la relégation, multipliant les incidents, les évasions, les revendications. Lorsque la commission de classement statuait sur un détenu évadé, l'acte même lui valait cette catégorisation, à plus forte raison lorsqu'il avait commis un nouveau délit avant de se faire reprendre.

\section{APPLIQUER DES MODÈLES D'OBSERVATION}

Les synthèses des éducateurs des centres d'observation ou de semi-liberté sont toutes rédigées sur un même modèle que Pierre Cannat a professé lors de ses cours au centre d'études de Fresnes ${ }^{23}$. Il s'inspire des recommandations de la Commission internationale pénale et pénitentiaire qui, en 1937, avait proposé un dossier réunissant toutes les données sur un individu et du formulaire général mis en place en Belgique. Il apporte des précisions et des exemples dans plusieurs revues. Ainsi en 1950, dans la Revue de science criminelle et de droit pénal comparé, il décrit «un type de relégué ${ }^{24}$. C'est en fait une synthèse d'observations effectuées à Loos-lèsLille. Après avoir évoqué longuement l'enfance, la jeunesse et la délinquance de l'individu, il décrit le physique, puis l'intelligence, le caractère et l'attitude. Ensuite il analyse son comportement dans le centre de triage et livre son pronostic. Dans ce cas, il est sombre: «M... est destiné à croupir de cellule en cellule, de cachot en cachot. Il est bouleversant de penser à un tel gaspillage d'âme humaine. La médecine ou la chirurgie nous livreront-elles quelque jour le secret d'une thérapie de l'anarchie constitutionnelle?» L'approche physique, anthropométrique est donc recommandée. Pierre Cannat écrit: «Passe pour le physique. Relativement petit, maigrichon $(58 \mathrm{~kg})$; le visage tourmenté, osseux, avec des yeux vifs, le sujet est nerveux et présente des tics de la face. Syphilitique comme de juste. L'auscultation révèle une légère rétractation de l'hémithorax droit, suite d'une pleurésie ou d'une pleurite ancienne. L'homme porte plutôt 22 ou 24 ans que 29. C'est un chétif. Il a des vertiges et des migraines, surtout le matin».

22 Jean Dublineau, médecin des hôpitaux psychiatriques de la Seine, 1949.

23 La douzième leçon du cours publié par Pierre Cannat en 1949 est consacrée à l'observation du détenu. Il y reproduit le formulaire belge. Cannat (1949b).

24 Cannat (1950a). 
En 1951, il présente dans la Revue pénitentiaire et de droit pénal une fiche criminologique utilisée à titre d'essai dans les maisons centrales de Melun et de Haguenau pour les détenus soumis au régime progressif. Les rubriques principales sont les suivantes: facteurs physiques, facteurs psychologiques, facteurs sociaux, corrélations et perspectives d'avenir. La même année, il livre un second portrait de relégué hébergé au centre de Lille à la Revue de science criminelle et de droit pénal comparé: «C'est un petit homme maigre et chétif, la peau du visage tendue sur un masque osseux à gros reliefs : zigomas proéminents, prognathisme supérieur accentué, des yeux doux et intelligents légèrement clos. Il fait penser aux chats de gouttières $»^{25}$.

L'examen médico-psychologique et social étant au cœur de la criminologie de l'après-guerre, il n'est pas étonnant de voir se multiplier les recommandations sur les méthodes à employer. Jean Pinatel s'y emploie en 1953 et décrit précisément ses différentes phases: enquête sociale, examen médical, examen psychiatrique, examen psychologique. Le développement physique du sujet doit permettre de «constater certains stigmates de dégénérescence, certains signes d'hérédo-syphilis, de déterminer l'état de santé actuel et de le juger en fonction des antécédents héréditaires et personnels $\gg^{26}$.

En 1955, Pierre Cannat présente dans la Revue pénitentiaire vingt portraits de relégués du centre lillois stabilisés en liberté conditionnelle ${ }^{27}$. Il résume leurs dossiers qui comportent presque tous des mentions sur le physique: "défiguré par un coup de rasoir reçu aux Bat' d'Af'», «déficience physique, amaigri. A été soigné pour tuberculose pulmonaire en 1943 », «tatouage sur le thorax», «marqué par la vie de prison», «paraît vieilli, assez tassé», «gros stigmates dégénératifs », «de taille moyenne, en parfaite santé, le sujet âgé de 30 ans semble jouir d'une grande vitalité», «allure de dur», «petite corpulence, extrémités courtes, gros, peu robuste», «sujet paraissant plus que son âge», «méridional assez maniéré, trop poli, grimaces, mimique vaguement hilare», «ne paraît pas marqué physiquement par la prison bien qu'il le soit moralement», «aspect rude», «tatouage sur les membres supérieurs», «sujet jeune de constitution robuste, mais d'aspect mou et avachi», «tatouages sur le tronc et les membres supérieurs; dentition en mauvais état», «tatouages sur le tronc et les membres supérieurs».

La même année, André Perdriau, contrôleur général des services pénitentiaires, reproduit dans la Revue de science criminelle le schéma type d'une enquête sociale: milieu familial d'origine, vie du détenu et comportement, personnalité du détenu, antécédents judiciaires, antécédents pathologiques, état de santé de la descendance, avenir, conclusion avec cette ultime question: «Que faudrait-il faire pour éviter la récidive $? \gg^{28}$.

Les fiches biographiques rédigées par les éducateurs des centres de tri comportent donc toujours une rubrique sur les aspects anthropologiques du sujet augmentée de mentions sur les stigmates psycho-physiologiques et les anomalies psychiques. Le tout est accompagné de jeux de photographies anthropométriques de face et de profil. On peut lire en 1955 à Rouen à propos d'un relégué de petite taille: «C'est

\footnotetext{
25 Cannat (1951).

26 Pinatel (1953).

27 Cannat (1955).

28 Perdriau (1955).
} 
presque un nain: grosse tête et membres courts. Les yeux gris ardoisés; des cheveux châtains; un front étroit. Le nez retroussé dévié sur la gauche. Prognathisme des deux mâchoires, menton fuyant. Trois doigts de la main gauche déformés. Assymétrie faciale. Testicule gauche atrophié. Très onicophage. Pas de tatouages et pas d'énurésie ${ }^{29}$. Ou encore ceci, en 1958: «Les yeux moyennement enfoncés dans l'orbite sont grands, ronds; les paupières supérieures sont relevées; il n'y a pas de poches sous les yeux. L'iris est marron, le profil fronto-nasal est brisé, tandis que le naso-bucal est légèrement prognathe. Le nez est légèrement busqué à base légèrement relevée, son dos est large et son bout gros $»^{30}$.

\section{USURE ET EMPREINTE PÉNITENTIAIRE}

En grande majorité, les relégués sont des hommes dans la force de l'âge au moment où ils sont frappés de cette peine complémentaire. Il est cependant permis de mentionner une usure physiologique des relégués. Dans les dossiers reviennent les expressions «état physique déficient», «sujet diminué par son état physique général», «terrain anémique et souffrant de graves affections »... Les observateurs signalent des hommes qui paraissent plus vieux que leur âge.

En 1949, sur 282 relégués étudiés à Saint-Martin-de-Ré, 36 ont été condamnés avant 30 ans, 127 avant 40 ans $^{31}$. Nous avons pu identifier 459 relégués admis au centre salutiste de Radepont dans l'Eure. Près de $70 \%$ ont été condamnés à la relégation avant l'âge de 40 ans. Mais antérieurement à leur hébergement dans cette œuvre de «relèvement par le travail», $21 \%$ ont séjourné entre 15 et 27 ans dans les centres de relégués, $31 \%$ entre 10 et 14 années $^{32}$.

Tableau 2. Âge des condamnés lors du prononcé de la relégation (analyse de 203 connus sur les 459 identifiés à Radepont).

\begin{tabular}{|c|c|c|}
\hline Âge & Effectif & Pourcentage \\
\hline Moins de 30 ans & 54 & $26,5 \%$ \\
\hline $30-34$ ans & 46 & $22,5 \%$ \\
\hline $35-39$ ans & 37 & $18,5 \%$ \\
\hline $40-44$ ans & 34 & $17 \%$ \\
\hline $45-49$ ans & 34 & $17 \%$ \\
\hline $50-54$ ans & 10 & $5 \%$ \\
\hline $55-59$ ans & 3 & $1,5 \%$ \\
\hline $60-64$ ans & 2 & $1 \%$ \\
\hline
\end{tabular}

29 Archives départementales de Seine-Maritime, Dossiers d'observation de relégués, 3735 W 1.

30 Ibidem.

31 Finelli, inspecteur de l'administration, 1951.

32 Les données chiffrées proviennent des archives conservées au château de Radepont: dossiers personnels et fichier des sorties. Voir Vimont, 2005. 
Tableau 3. Âge des relégués lors de leur arrivée à Radepont (analyse de 429 connus sur les 459 identifiés à Radepont).

\begin{tabular}{|c|c|c|}
\hline Âge & Effectif & Pourcentage \\
\hline Moins de 30 ans & 7 & $1,5 \%$ \\
\hline $30-34$ ans & 28 & $6,5 \%$ \\
\hline $35-39$ ans & 48 & $11 \%$ \\
\hline $40-44$ ans & 76 & $18 \%$ \\
\hline $45-49$ ans & 84 & $19,5 \%$ \\
\hline $50-54$ ans & 74 & $17 \%$ \\
\hline $55-59$ ans & 61 & $14 \%$ \\
\hline $60-64$ ans & 38 & $9 \%$ \\
\hline $65-69$ ans & 10 & $2,5 \%$ \\
\hline $70-74$ ans & 3 & $0,5 \%$ \\
\hline
\end{tabular}

Tableau 4. Temps écoulé entre le prononcé de la relégation et la date d'arrivée à Radepont (analyse de 230 connus sur les 459 identifiés à Radepont).

\begin{tabular}{|c|c|c|}
\hline Années & Effectif & Pourcentage \\
\hline De 1 an à 4 ans & 28 & $12 \%$ \\
\hline De 5 à 9 ans & 81 & $35 \%$ \\
\hline De 10 ans à 14 ans & 71 & $31 \%$ \\
\hline De 15 à 19 ans & 31 & $13,5 \%$ \\
\hline De 20 ans à 27 ans & 17 & $7,5 \%$ \\
\hline
\end{tabular}

Au centre de semi-liberté de Clermont-Ferrand, l'échantillon de 266 dossiers étudiés confirme les pourcentages précédents, même si les hôtes sont un peu plus jeunes que ceux de Radepont. Dans les deux sites, $50 \%$ des prisonniers ont subi plus de dix années de relégation.

Tableau 5. Âge des condamnés lors du prononcé de la relégation (analyse de 255 connus sur les 266 identifiés à Clermont-Ferrand).

\begin{tabular}{|c|c|c|}
\hline Âge & Effectif & Pourcentage \\
\hline Moins de 30 ans & 83 & $32,5 \%$ \\
\hline $30-34$ ans & 56 & $22 \%$ \\
\hline $35-39$ ans & 51 & $20 \%$ \\
\hline $40-44$ ans & 32 & $12,5 \%$ \\
\hline $45-49$ ans & 23 & $9 \%$ \\
\hline $50-54$ ans & 10 & $4 \%$ \\
\hline
\end{tabular}


Tableau 6. Âge des relégués lors de leur arrivée à Clermont-Ferrand (analyse de 201 connus sur les 266 identifiés à Clermont-Ferrand).

\begin{tabular}{|c|c|c|}
\hline Âge & Effectif & Pourcentage \\
\hline Moins de 30 ans & 4 & $2 \%$ \\
\hline $30-34$ ans & 22 & $11 \%$ \\
\hline $35-39$ ans & 28 & $14 \%$ \\
\hline $40-44$ ans & 52 & $16 \%$ \\
\hline $45-49$ ans & 32 & $17,5 \%$ \\
\hline $50-54$ ans & 35 & $10,5 \%$ \\
\hline $55-59$ ans & 21 & $3,5 \%$ \\
\hline $60-64$ ans & 7 & \\
\hline $65-69$ ans & & \\
\hline $70-74$ ans & &
\end{tabular}

Tableau 7. Temps écoulé entre le prononcé de la relégation et la date d'arrivée à Clermont-Ferrand (analyse de 200 connus sur les 266 identifiés à Clermont-Ferrand).

\begin{tabular}{|c|c|c|}
\hline Années & Effectif & Pourcentage \\
\hline De 1 an à 4 ans & 13 & $6,5 \%$ \\
\hline De 5 à 9 ans & 87 & $43,5 \%$ \\
\hline De 10 ans à 14 ans & 49 & $24,5 \%$ \\
\hline De 15 à 19 ans & 41 & $20,5 \%$ \\
\hline De 20 ans à 27 ans & 10 & $5 \%$ \\
\hline
\end{tabular}

Les longs emprisonnements dans les prisons et les centres ont porté atteinte à leur intégrité corporelle. Certains ont connu la misère et les privations des maisons centrales pendant l'Occupation. En 1954, un relégué est évalué au centre de Rouen. Il vient de subir 22 années de détention et a séjourné à Fontevraud pendant la guerre. Sa vue est très faible $\left(2 / 10^{\mathrm{e}}\right)$. Le psychiatre signale qu'il est «précocement vieilli » (il n'a que 48 ans) et que son état physique est déficient. Il conclut son rapport en le qualifiant de «déchet social» ${ }^{33}$. Un multirécidiviste du vol de bicyclettes - quinze condamnations avant la relégation prononcée en 1942 - arrive dans le même centre en 1958. Il traîne de prison en prison depuis vingt-cinq ans. Sa fiche psychiatrique le décrit ainsi: «Pauvre type pessimiste à l'allure lymphatique, débilard ${ }^{34}$. À Lille, en 1952, l'éducateur note à propos d'un détenu qui vient d'achever 33 années de détention: «Ancien antisocial tassé et très diminué par l'âge et l'empreinte pénitentiaire». La commission de classement range cet homme de 53 ans parmi les asociaux ${ }^{35}$. Le

33 Archives départementales de Seine-Maritime, Dossiers d'observations de relégués, 3735 W 2.

34 Ibidem.

35 Archives départementales du Puy-de-Dôme, 1124 W 267, Dossier de Lucien R. Rapport de synthèse établi à Loos-lès-Lille en 1952. 
comportement des hôtes des centres d'observation met en relief cette usure pénitentiaire: "Allure lente, lasse, désabusée, paresseuse; il déambule, les mains au fond des poches, les épaules remontées, ne se pressant jamais. Il a pris le parti de sa dégradation et indique avec fatalisme: «nous les relégués, on n'est pas grandchose $»^{36}$.

Les mentions de handicaps, d'infirmités ne sont pas rares et contribuent à des pronostics défavorables sur le reclassement. Quelques notations dans les dossiers rouennais et clermontois: insuffisance cardiaque, jambe raide depuis 1942, bras droit atrophié, chétif, malingre, moyens physiques réduits, parkinsonien ayant subi une lobotomie préfrontale qui a échoué et qui le laisse «passablement abruti»...

Les blessures de guerre accroissent les difficultés, car les destinées de la plupart de ces hommes furent brutalisées par le second conflit mondial. Il est question de traumatisme céphalique lié à un éclat de bombe, d'une fracture du crâne valant pension de $20 \%$ d'invalidité pour un ancien des brigades internationales et de la légion étrangère, d'un visage défiguré par les blessures reçues en 1940, d'une santé fragile suite à un engagement militaire en Indochine, de l'amputation et de la pension d'un tenancier de bar, incarcéré à Clairvaux pendant l'Occupation, transféré à Belle Isle pour ferrailler à la construction de l'Atlantique, sous les coups de crosse des gardiens. Les dossiers rappellent que des droits communs ont été extraits des maisons centrales pour être déportés dans des camps en Allemagne d'où ils revinrent diminués.

La tuberculose est en passe de guérison, mais nombre de relégués ont fait de longs séjours au sanatorium de Liancourt. Les traitements les handicapent durablement. L'un a eu sept côtes d'enlevées et on lui a pratiqué deux thoracos. Il a cinquante-trois ans lors de son arrivée au centre Pélissier, il est décrit comme diminué physiquement ${ }^{37}$. Alexandre $\mathrm{S}$. a cinquante et un ans lorsqu'il est observé à Besançon en 1954. Il a derrière lui dix-neuf années de détention, après une condamnation à vingt années de travaux forcés. Pendant deux années, il a été soigné pour une tuberculose pulmonaire à Liancourt. Son dossier le présente comme excessivement marqué par sa détention, malade et incapable de travailler. Il paraît soixante ans ${ }^{38}$. L'hôpital-prison de Liancourt soigne, mais l'alcool y fait des ravages, comme l'a montré Alphonse Boudard ${ }^{39}$. Pour certains tuberculeux, l'aide du centre de Radepont était indispensable.

Les alcooliques sont marqués, diminués et les observateurs ne manquent pas de le signaler. Les mentions «faciès d'ivrogne», «épave», «clochard alcoolique» ne sont pas rares. C'est le cas de Jean V., relégué à quarante-trois ans en 1952. Il a derrière lui dix années d'engagement dans les troupes coloniales au Maroc et en Indochine où il a contracté des habitudes d'intempérance. Il est passé par le centre de Rouen en 1956, d'où il a été exclu pour ivresses répétées et bagarres avec d'autres ivrognes, par le centre de semi-liberté Boudet en 1961, d'où il est exclu pour ivresse,

36 Archives départementales de Seine-Maritime, Dossiers d'observations de relégués, 3735 W 3.

37 Archives départementales du Puy-de-Dôme, 1124 W 268, Dossier de Fernand T. Rapport de synthèse établi à Clermont-Ferrand le 5 novembre 1965 .

38 Archives départementales du Puy-de-Dôme, 1124 W 268, Dossier de Alexandre S. Dossier établi au COR de Besançon en 1954.

39 Plusieurs ouvrages d'Alphonse Boudard évoquent ce sanatorium pénitentiaire. Voir Boudard, 2000, p. 167. 
par le centre de Clermont-Ferrand, d'où il est exclu en 1965 après une bagarre entre ivrognes. Cette ultime frasque lui coûte deux ans de stage disciplinaire dans la maison centrale de Poissy. Il n'en sort qu'en 1967, après quinze années de relégation, «précocement vieilli $»^{40}$.

Les échecs répétés en liberté conditionnelle allongent considérablement les séjours des relégués dans l'univers carcéral. Les éducateurs y voient l'une des causes des comportements résignés, des allures nonchalantes, de l'absence de volonté. «Marqué par l'empreinte pénitentiaire », «bien intégré au milieu carcéral », «aimable clochard qui ne peut vivre qu'en milieu fermé» désignent ceux qui, employés au service général, ne parviennent pas à affronter la liberté, rechutent dans l'alcool à la moindre occasion ou commettent de menus larcins. Dès 1950, le docteur Vullien, médecin-chef à l'asile d'Armentières, tire un premier bilan de l'expérience d'observation lilloise. Il a examiné les cent premiers relégués admis dans la maison centrale. Il souligne un trait dominant, l'empreinte pénitentiaire: «À force de vivre en prison, ils se sont adaptés à un monde nouveau concentrationnaire dont ils ont épousé les coutumes. Pliés à la discipline, à l'obéissance, ils ont pris l'habitude d'une vie stéréotypée, minutée, inférieure, mais exempte des soucis qui assaillent à chaque instant l'homme libre; contraints de dissimuler leurs mauvais penchants, on les voit mendier sans vergogne les bonnes grâces des surveillants et des supérieurs, se préoccuper sans pudeur de la satisfaction immédiate de leurs besoins matériels. (...) Inutile d'insister sur le rôle néfaste de la masturbation (qui est de règle) et de la pédérastie malheureusement trop fréquente ${ }^{41}$. Le $\mathrm{II}^{\mathrm{e}}$ Congrès international de criminologie, organisé à Paris en septembre 1950, s'efforce de répondre à la question: «La prison est-elle un facteur criminogène?». Les spécialistes des questions pénitentiaires s'y disent frappés par l'existence d'un type « détenu». Ils l'expliquent par un changement de l'habitus lié à la rigidité disciplinaire, à l'automatisme de la vie carcérale, au port du droguet. Ils relient cette approche physique à des traits psychologiques: le mensonge, l'hypocrisie et la dissimulation.

En 1961, l'éducateur du centre Pélissier à Clermont-Ferrand conclut ainsi une synthèse sur un ancien des maisons de correction et des Bat' d'Af': "Très intégré au milieu carcéral, il reconnaît d'ailleurs que la Pénitentiaire est sa seule famille. Il a un tempérament de valet de chambre, aime le contact avec le personnel, se dévoue volontiers. Il est heureux quand il a une place au service général et donne alors toute satisfaction. Il ne veut pas sortir de prison ${ }^{42}$. Également en 1961, cette appréciation sur un berger corse qui entame sa dixième année de relégation: "Le type même du pilier de prison, taulard par prédestination et par goût, très adapté aux détentions $»^{43}$. Trois ans plus tard, le même éducateur à propos d'un autre relégué: "C'est une épave sociale qui a trouvé son équilibre en prison $»^{44}$.

40 Archives départementales du Puy-de-Dôme, 1124 W 268, Dossier de Jean V. Rapport de synthèse établi à Clermont-Ferrand le 30 octobre 1962.

${ }^{41}$ Vullien (1950, p. 35).

42 Archives départementales du Puy-de-Dôme, 1124 W 268, Dossier de Jean V.-M. Rapport de synthèse établi à Clermont-Ferrand le 31 janvier 1961.

43 Archives départementales du Puy-de-Dôme, 1124 W 268, Dossier de Jules U. Rapport établi au centre Pélissier le 15 mai 1961.

44 Archives départementales du Puy-de-Dôme, 1124 W 268, Dossier de André V. Rapport de synthèse établi à Clermont-Ferrand en 1964. 
$\mathrm{Du}$ «type détenu»au «relégué type» marqué par l'empreinte pénitentiaire, il n'y a qu'un pas que les observateurs franchissent fréquemment. C'est un récidiviste chevronné, un revendicateur, un paresseux qui se sent injustement persécuté. Il est sournois, irrécupérable. Certains rapports regroupent ces traits au sein d'une «psychose classique du relégué».

\section{LE NÉO-LOMBROSISME}

Il peut sembler étonnant de lire pendant les années cinquante et au début des années soixante des appréciations directement inspirées des théories de César Lombroso sous la plume d'éducateurs et de psychiatres. L'éducateur du centre Pélissier de Clermont-Ferrand écrit en juin 1960: «C'est un délinquant comme les décrit Lombroso ${ }^{45}$. En juillet 1961, le même éducateur: «Sur le plan criminologique, le cas de P. est très attachant; il s'agit d'un véritable délinquant-né » ${ }^{46}$. Un relégué d'origine gitane est qualifié de «fugueur-né par déséquilibre organique et par prédisposition ethnique ${ }^{47}$. Un cambrioleur, classé parmi les antisociaux au centre de Besançon en 1959, est présenté en 1965 à Clermont comme un «casseur» au «comportement typique de dur» avec cette appréciation supplémentaire: «Antisocial par conviction et par une tare organique congénitale. Exemple typique du délinquant-né».

Pierre Grapin avait souligné, en 1949, le réveil de certaines idées lombrosiennes, enrichies par l'apport de théories psychopathologiques, dans les recherches d'anthropologie criminelle $\mathrm{e}^{48}$. Le deuxième tome des actes du II $^{\mathrm{e}}$ Congrès international de criminologie consacré à la biologie et à l'anthropologie en témoignait également ${ }^{49}$. Les néo-lombrosiens continuaient de considérer que le crime avait un caractère pathologique et repéraient des stigmates anatomiques, morphologiques, neurologiques, fonctionnels. Ils tentaient de mettre en valeur des biotypes criminels repérables par des surracentuations constitutionnelles ou des altérations individuelles héréditaires ou acquises.

\section{Frustes et brutes}

Si le simple coup d'œil ne suffit plus à étiqueter les hôtes des centres, des mentions assez succinctes résument l'impression des observateurs. Un garçon de ferme breton, issu d'un milieu «fruste» de pêcheurs est qualifié de «bonne brute » à l'âge mental de neuf ans par le psychiatre du centre d'observation de Loos-lès-Lille, le docteur Vullien ${ }^{50}$. Un berger corse est présenté comme un «sujet fruste, débile men-

45 Archives départementales du Puy-de-Dôme, 1124 W 256, Dossier de Jean A. Rapport de synthèse établi à Clermont-Ferrand en juin 1960.

46 Archives départementales du Puy-de-Dôme, 1124 W 266, Dossier de Paul P. Rapport de synthèse établi à Clermont-Ferrand le 30 juillet 1961.

47 Archives départementales du Puy-de-Dôme, 1124 W 268, Dossier de Lucien V. D. H. Rapport établi à Clermont-Ferrand le 30 juillet 1960 par l'éducateur en charge du centre Pélissier.

48 Grapin (1949).

49 Collectif (1952).

50 Archives départementales du Puy-de-Dôme, 1124 W 268, Dossier de André V. Rapport établi au COR de Lille en décembre 1951. 
tal, impulsif et coléreux $»^{51}$ par le psychiatre Benoiston au centre de triage de Rouen en 1956. Les avis sont proches sur un ouvrier agricole lorrain, récidiviste des attentats à la pudeur sur des garçonnets. À Rouen en 1960, le psychiatre Corre le présente comme un sujet fruste. À Clermont-Ferrand en 1964, il est décrit comme fruste et bestial. Un manœuvre alsacien, classé antisocial à Lille à cause de ses réactions violentes sous l'empire de l'alcool est décrit comme un excellent élément très robuste dans la prison de Gannat, puis comme «une grosse brute alcoolique» lors de son placement en semi-liberté à Clermont-Ferrand.

\section{Stigmates dégénératifs}

Les dossiers d'observation offrent des synthèses biographiques alimentées par les enquêtes menées par les assistantes sociales de la région d'origine du relégué, par les autobiographies exigées dans les centres, par les entretiens et observations menées par les éducateurs, les gardiens et les psychiatres. Les antécédents familiaux et le repérage de maladies chez les ascendants sont scrupuleusement notés. Le lien entre hérédité et criminalité est encore patent pour une grande partie des criminologues, comme le montre un article de Jean Pinatel, "Hérédité et criminalité», publié en 1954. Nombre d'observateurs des centres de relégués se réfèrent implicitement aux théories de Morel et Magnan sur la dégénérescence. L'alcoolisme de l'un ou de l'autre des parents conduit immanquablement à la classification d'aboulique alcoolique lorsque le sujet est revenu de sorties-promenades en état d'ivresse manifeste. «Hérédo-alcoolique ${ }^{52}$, «stigmates évidents d'alcoolisme héréditaire ${ }^{53}$, «signes dégénératifs d'alcoolisme héréditaire ${ }^{54}$, «dégénéré hérédosyphilitique et hérédoalcoolique $»^{55}$, «victime de tares organiques qui prédisposent à la délinquance», «signes dystrophiques évidents de syphilis congénitale (des malformations dentaires)» sont des mentions fréquentes dans les dossiers de synthèse établis par l'éducateur du centre de semi-liberté Pierre Giscard qui recevait des asociaux urbains et, fréquemment, des relégués ayant des problèmes avec l'alcool. En 1962, il note: «Dégénéré, il a mis au monde des dégénérés qui ont déjà mis au monde des dégénérés... et ainsi se perpétue la race ${ }^{56}$. Mais il n'est pas le seul éducateur à insister sur les supposées «tares familiales». En 1963, le psychiatre du centre de Rouen mentionne «un faciès un tantinet dégénératif ${ }^{57}$ et il conclut son rapport ainsi: «Tout cet ensemble est consternant et dans sa qualité et dans son agencement».

51 Archives départementales du Puy-de-Dôme, 1124 W 268, Dossier de Jules U. Rapport établi au COR de Rouen en 1956.

52 Archives départementales du Puy-de-Dôme, 1124 W 268, Dossier de Daniel V. Rapport de synthèse établi à Clermont-Ferrand le 30 juin 1959. L'éducateur Perrot prit ses fonctions en 1958 et demeura au centre jusqu'à la suppression de la relégation en 1970.

53 Archives départementales du Puy-de-Dôme, 1124 W 268, Dossier de Pierre V.-M. Rapport de synthèse établi à Clermont-Ferrand le 30 janvier 1961.

54 Archives départementales du Puy-de-Dôme, 1124 W 268, Dossier de Fernand T. Rapport de synthèse établi à Clermont-Ferrand le 5 novembre 1965.

55 Archives départementales du Puy-de-Dôme, 1124 W 268, Dossier de Jules U. Rapport établi au centre Pélissier le 15 mai 1961.

56 Archives départementales du Puy-de-Dôme, 1124 W 266, Dossier de André N. Rapport établi au centre Pélissier le 30 mai 1962.

57 Archives départementales de Seine-Maritime. Dossiers d'observation des relégués, 3735 W 1. 


\section{Dysmorphismes}

Les dossiers individuels comportent nombre de mentions d'anomalies morphologiques accusées que les évaluateurs lient à la récidive de délinquance. S'ils ne s'attardent pas sur la forme des crânes, les traits du visage retiennent fréquemment l'attention: prognathisme facial, saillie des arcades sourcilières, menton fuyant, front étroit et bas. Pierre Grapin, membre de la Société d'Anthropologie de Paris, avait pourtant insisté, dès 1954, sur l'absence de corrélations décisives entre dysmorphisme et criminalité ${ }^{58}$. Les éducateurs sont attentifs aux tailles «anormales» de certains relégués. L'un d'eux est décrit ainsi à Rouen: "Chétif, presque un nain, $1 \mathrm{~m} 45$, avec une grosse tête et des membres courts ${ }^{59}$. Les notations n'évoluent guère au fil des années. En 1959, l'éducateur de Clermont décrit ainsi un petit homme: "C'est un gnome de type pygmée avec des membres courts déformés et noueux $»^{60}$. Un autre a fait l'objet d'une expertise mentale à Poitiers en 1954: «C'est un individu de type longiligne, dysmorphique, sa voix est aiguë, son faciès piriforme est quelque peu simiesque ${ }^{61}$. Dix ans plus tard, à Rouen, un relégué est décrit ainsi: «Contenu et contenant assez clownesques, faciès dysmorphique ${ }^{62}$.

\section{Stigmates de troubles psychiques}

Le repérage des asociaux par les éducateurs qui rédigeaient les synthèses s'inspirait fortement des théories du docteur Bachet, médecin psychiatre dans le quartier des jeunes détenus de Fresnes et ancien psychiatre du patronage Rollet. Il livra en 1949 les résultats d'une étude médico-psychologique sur 47 relégués de passage à Fresnes. Il s'efforça, au cours d'entretiens, de repérer les liens entre les antécédents de dissociation familiale, des névroses telles que l'onycophagie, le somnambulisme, l'énurésie, les troubles de la parole ${ }^{63}$ et la fréquence des récidives de vols ${ }^{64}$. En 1950, il publia un ouvrage ${ }^{65}$, dans lequel il expliquait la genèse d'une part importante de la criminalité par un déficit des contrôles supérieurs lié à l'absence d'une séparation complète entre l'état de sommeil et l'état de veille. Il s'agissait principalement de la délinquance liée au déficit de volonté, à l'aboulie, au caractère instinctif des délits, à la mythomanie et au dédoublement de la personnalité. Les signes cliniques de ce trouble figurent dans les rapports de synthèse des éducateurs qui accordent une large place à l'attitude des relégués lors de la phase d'isolement cellulaire: difficulté à fixer l'attention, instabilité, prédominance des réactions émotives et colériques, énurésie prolongée et somnambulisme. Par ailleurs dans les fiches biographiques, les éducateurs notent l'ambidextrie, les tics, l'ectopie, l'énurésie, l'onycophagie, les asymétries faciales, le somnambulisme, les céphalées, les pertes de connaissance.

\footnotetext{
58 Grapin (1954).

59 Archives départementales de Seine-Maritime, Dossiers d'observation de relégués, 3735 W 1.

60 Archives départementales du Puy-de-Dôme, 1124 W 266, Dossier de Joseph P.

${ }_{61}$ Archives départementales de Seine-Maritime, Dossiers d'observation de relégués, 3735 W 4.

${ }^{62}$ Archives départementales de Seine-Maritime, Dossiers d'observation de relégués, 3735 W 2.

63 Dans un autre article, le docteur Bachet livre les résultats d'une étude menée avec une assistante sociale sur 760 détenus dont des relégués (1951).

64 Bachet (1949).

65 Bachet (1950).
} 


\section{Stéréotypes sur les «délinquants constitutionnels ethniques»}

Les dossiers d'observation et de suivi regorgent de préjugés et de stéréotypes sur les relégués issus de l'univers des gens du voyage, forains identifiés comme gitans, romanichels ou manouches. Ces hommes auraient une délinquance spécifique liée à leur origine, aux traditions de leur groupe. Instabilité, fugues, trafics et vols d'habitude, prostitution des membres de la famille ${ }^{66}$, usage du couteau, seraient des traits distinctifs de ces communautés, selon les avis des observateurs, tant à Saint-Martinde Ré qu'à Mauzac, Rouen ou à Clermont-Ferrand. Les avis sont tranchés, témoignent de nombreuses réticences à l'égard des gens du voyage et même d'un racisme latent. Un Yenich d'origine alsacienne est l'objet de deux appréciations successives. À Besançon, en 1958: «Milieu d'origine taré. Il s'agit de romanichels pour lesquels le vol n'est pas considéré comme un délit». À Clermont-Ferrand, en 1966: «Sa délinquance est celle d'un manouche. Elle est d'ordre ethnique et liée à la mentalité et aux conceptions tribales du sujet qui, comme tous ceux de sa race ne pourra jamais concevoir qu'il y ait une autre manière de se comporter que celle qu'il a connue jusqu'à présent ${ }^{67}$. Lucien V. D. H. est décrit ainsi: «Il est nettement marqué par ses origines ethniques et a un physique de levantin ou de sémite. Des manouches, il a également l'instabilité ethnique qui en fait un élément incapable de se fixer ${ }^{68}$. Une fiche sommaire établie à Saint-Martin en 1953 s'étonne de l'ardeur au travail d'un relégué, «malgré ses origines gitanes». À Mauzac, en 1950, un vannier qui a comparu neuf fois au prétoire est qualifié ainsi: «État d'esprit spécial au milieu forain». Le psychiatre de Rouen note en 1953 à propos d'un gitan: «Déchet social victime de ses origines». On peut lire également quelques stéréotypes sur les méditerranéens. C'est par exemple le cas d'un relégué d'origine sicilienne, qualifié de délinquant ethnique prédisposé au métier de souteneur ${ }^{69}$.

\section{CONCLUSIONS LAPIDAIRES ET CLASSEMENTS SOMMAIRES}

Nous avons isolé les unes des autres les différentes formes de description du physique des relégués. Elles ne doivent pas être séparées des appréciations psychiatriques et psychologiques. L'influence de l'Italien Di Tullio ${ }^{70}$ se remarque par les nombreuses mentions de «délinquants constitutionnels» et celle du Français

66 Rapport établi à Clermont-Ferrand le 30 avril 1960: «Sa délinquance ethnique correspond à la mentalité et aux habitudes des tribus de «voyageurs » dont C. a gardé la mentalité. Il est normal, pour ces gens-là, que les femmes de la tribu se prostituent, à condition évidemment qu'il ne s'agisse que de «travail», tout sentimentalisme doit être exclu». Archives départementales du Puy-de-Dôme, 1124 W 258.

${ }^{67}$ Archives départementales du Puy-de-Dôme, 1124 W 256, Dossier de Jean A. Rapports établis en 1958 au centre de tri de Besançon et en 1965 au centre Pélissier de Clermont-Ferrand.

68 Archives départementales du Puy-de-Dôme, 1124 W 268, Dossier de Lucien V. D. H. Rapport de synthèse établi à Clermont-Ferrand le 30 juillet 1960.

69 Archives départementales du Puy-de-Dôme, 1124 W 264, Dossier de Marcel L. Rapport de synthèse établi à Clermont-Ferrand le 4 juin 1965: «Les Siciliens ont une mentalité spéciale, faite d'une activité fruste et déviée, axée sur l'honneur familial, ce qui n'empêche pas les frères et les sœurs de coucher ensemble et qui prédispose comme pour la majorité des méditerranéens à la carrière de souteneur».

70 Di Tullio (1951). 
Dupré $^{71}$ à propos des «pervers instinctifs ». Lors des entretiens menés par les éducateurs, l'allure du relégué est également appréciée ou stigmatisée: "allure de caïd», «abord franc et direct», «dissimulé et sournois», «allure très homosexuelle ${ }^{72}$. Quelques malchanceux accumulent des notations appartenant aux différents registres que nous avons évoqués.

C'est le cas d'un marin, évalué en 1957 à Clermont-Ferrand: «Il appartient à une famille de marins-pêcheurs bretons. Des gens honnêtes, mais un milieu où l'alcoolisme règne en maître. Fortement dystrophique, faciès brutal et tourmenté, il a des troubles de la parole. Il y a donc à la base de son déséquilibre une tare qui a conditionné sa délinquance et le poivrot impénitent qu'il est aujourd'hui. Délinquant constitutionnel en raison de sa dégénérescence et de son hérédité alcoolique, je ne l'ai jamais vu à jeun, même le matin. C'est un parasite incapable de concevoir et de mener une vie normale, qui ne doit sa liberté actuelle qu'à la trop grande mansuétude du comité de Clermont-Ferrand $»^{73}$.

Un docker alcoolique, rangé parmi les antisociaux à Lille en 1959, est décrit ainsi quatre ans plus tard à Clermont-Ferrand: «Il porte des signes dystrophiques assez discrets mais évidents de syphilis et d'alcoolisme héréditaire. Il s'agit d'un maniaque intoxiqué. Ce sont là les plus dangereux, car les effets de l'ivresse se manifestent sur un fond de déséquilibre et de perversité lors de manifestations d'agressivité contre la société. En somme, un dégénéré, inéduqué et inéducable, épileptique, alcoolique dangereux, délinquant d'habitude, pervers et déséquilibré mental. Il semble qu'il y a là assez d'éléments pour qu'une société organisée ait le droit de réclamer une mesure de sûreté définitive contre lui ${ }^{74}$.

Elles sont combinées à des évaluations de leur intelligence par des tests, de leurs troubles psychiques par les psychiatres pour aboutir à des formules lapidaires qui concluent les rapports de synthèse soumis aux commissions de classement ou de mise en liberté. Bien souvent, elles sont accompagnées d'une proposition de l'auteur, suivie ou non par la commission.

Un récidiviste du port illégal d'uniforme et de décorations, ancien d'Indochine et de Corée, est qualifié en 1963 de «déséquilibré de type schizoïde, instable, alcoolique et toxicomane». Le pronostic est pessimiste: «Échec certain » ${ }^{75}$. Un «déséquilibré caractériel» est « voué à l'asile et non plus à la prison » ${ }^{76}$. La seule solution proposée à un «déchet humain, débile alcoolomaniaque » est une cure de désintoxication dans les prisons de Lyon ${ }^{77}$. D'autres ne sont pas «mûrs pour la liberté», sont

71 Dupré (1925).

72 L'éducateur de Clermont-Ferrand note le 30 mars 1960 à propos d'un vagabond: «Probablement pédéraste comme la majorité des relégués». Archives départementales du Puy-de-Dôme, $1124 \mathrm{~W}$ 267, Dossier de Marc R.

73 Archives départementales du Puy-de-Dôme, 1124 W 268, Dossier de Hippolyte S. Rapport de synthèse établi à Clermont-Ferrand en 1957.

74 Archives départementales du Puy-de-Dôme, 1124 W 268, Dossier de Robert S. Rapport de synthèse établi à Clermont-Ferrand le 30 mai 1963.

75 Archives départementales du Puy-de-Dôme, 1124 W 268, Dossier de André V. Rapport de synthèse établi à Clermont-Ferrand en 1964.

76 Archives départementales du Puy-de-Dôme, 1124 W 268, Dossier de Daniel V. Rapport de synthèse établi à Clermont-Ferrand le 30 juin 1959.

77 Archives départementales du Puy-de-Dôme, 1124 W 268, Dossier de Paul V. Rapport de synthèse établi à Clermont-Ferrand le 20 janvier 1959. 
«voués à l'état carcéral» car «désocialisés à jamais ». En 1965, un «casseur » parisien est dit «inamendé et inamendable». Encore plus sommaire cet avis sur un relégué de Clermont-Ferrand, ancien d'Indochine, fréquemment interné en hôpital pour ses tentatives de suicide: «Du reste, si par erreur (ou par chance) il arrivait à ne pas se rater, il accomplirait ainsi le seul acte socialement utile de sa vie de parasite».

Des divergences d'appréciation entre les éducateurs et les commissions de mise en libération conditionnelle sont perceptibles, même si énoncées avec mesure, pendant les années soixante lorsque s'estompe l'optimisme des initiateurs de la réforme pénitentiaire. Ainsi, à Clermont-Ferrand, en 1961: «Une réinsertion pour de tels hommes ressemble à une mauvaise plaisanterie». Également à Clermont-Ferrand, en 1965: «Il est le type même de ces relégués dont l'avenir est en prison et qu'il est absurde et dangereux de remettre en circulation. Nous faisons ainsi leur propre malheur, nous les vouons à la 'cloche', au froid, à la faim, et en dernière analyse, à la récidive. La solution humaine et équitable serait de lui assurer une vie détenue dans un établissement libéral mais fermé ${ }^{78}$. La «trop grande mansuétude» du comité de Clermont-Ferrand et du magistrat qui la préside est mise en cause par l'auteur des rapports de synthèse, l'éducateur du centre Pélissier qui écrit en 1965: «Opposition formelle (mais platonique hélas) à toute tentative de sortie».

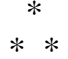

Les responsables de l'Administration pénitentiaire ont évalué à plusieurs reprises l'efficacité du dispositif mis en place à l'égard des relégués. Un tiers, voire un quart des pensionnaires des centres parvenaient à se stabiliser en liberté conditionnelle. Aux yeux de Pierre Cannat, ce n'était pas négligeable compte tenu des handicaps de ces prisonniers. Même avis de André Touren, directeur de l'Administration pénitentiaire en 1956, qui soulignait que la sentence indéterminée et la diversification des établissements étaient des armes parfaitement adaptées «aux faiblesses de la nature des intéressés ${ }^{79}$. Les deux tiers ou les trois quarts restants étaient voués au parcours carcéral sans cesse recommencé après les échecs en libération conditionnelle. Les plus chanceux parvenaient à s'évanouir dans la nature grâce à des certificats de complaisance qui leur permettaient d'obtenir une libération conditionnelle à partir des centres de Ré et de Mauzac sans passer par des centres de triage qui ne pouvaient pas tous les accueillir ${ }^{80}$. La semi-liberté dans les centres de Clermont-Ferrand, de SaintSulpice permettait un «atterrissage feutré», selon l'expression de Pierre Cannat. Elle ne présentait guère de risques, elle était une réponse au problème sexuel et entretenait « ces déchets sociaux dans une atmosphère mêlée de contrainte et de libéralisme correspondant à leur absence d'agressivité, à leur misère congénitale, et au devoir qu'a l'Administration de ne jamais tuer en eux l'espoir ${ }^{81}$. De tous temps les fuites

78 Archives départementales du Puy-de-Dôme, 1124 W 268, Dossier de Fernand T. Rapport de synthèse établi à Clermont-Ferrand le 6 novembre 1965.

79 Touren (1956).

80 En 1956, le chef du bureau de l'application des peines qui avait succédé à Pierre Cannat, M. Perdriau estimait qu'un sixième des relégués obtenaient une libération conditionnelle sans passer par les centres de triage; Perdriau, 1956, p. 897.

81 Cannat (1953, p. 332). 
en semi-liberté furent nombreuses et il semble que l'administration ne mit guère d'empressement à retrouver ceux qui ne commettaient plus de délits. Seule la suppression de la relégation en 1970 permit aux plus malchanceux de retrouver définitivement la liberté.

La confrontation entre les textes des promoteurs de la réforme pénitentiaire et les rapports d'observation effectués dans les centres de relégués montre un profond décalage entre la générosité des premiers, leur optimisme et leur pari dans l'amendement de l'homme et le pessimisme des éducateurs et des médecins du terrain, confrontés à tous les types de misère de ces lendemains de guerre. Il nous manque le point de vue des relégués eux-mêmes, intériorisant parfois les paroles critiques de leurs évaluateurs, se révoltant contre le principe même des stages d'évaluations à l'instar de quelques antisociaux ou trichant lors des entretiens et des tests pour franchir plus vite les étapes vers la libération conditionnelle ${ }^{82}$.

Les corps usés, estropiés, déformés, tatoués font sens et influencent classements et pronostics. Pour une large fraction des relégués, la criminologie appliquée est synonyme d'un allongement de la peine de la relégation par des orientations successives dans des établissements adaptés à leur personnalité. L'observation trie principalement pour mieux incarcérer, elle ne contribue qu'occasionnellement à la libération définitive.

Jean-Claude Vimont

Université de Rouen

Département d'histoire

1 , rue Thomas Becket

76821 Mont-Saint-Aignan Cedex jean-claude.vimont@wanadoo.fr

\section{BIBLIOGRAPHIE}

Ancel, M., Enquête sur les mesures de sûreté en matière criminelle, Melun, Imprimerie administrative, $1950 \mathrm{~b}$.

Ancel, M., La défense sociale nouvelle, Paris, Éditions Cujas, 1954.

Arnaud, G., Prisons 53, Paris, Julliard, 1953.

Bachet, M., Étude médico-psychologique concernant 47 délinquants récidivistes relégués, Revue de Science criminelle et de droit pénal comparé, 1949, 2, pp. 47-72.

Bachet, M., Les Encéphaloses criminogènes, Paris, Foucher, 1950.

Bachet, M., Rapport sur l'annexe psychiatrique des prisons de Fresnes, Revue pénitentiaire et de droit pénal, 1951, 3, pp. 658-732.

Boudard, A., La Cerise, Paris, La Table ronde, édition 2000.

Cannat, P., Nos frères les récidivistes, Esquisse d'une politique criminelle fondée sur le reclassement ou l'élimination des délinquants, Paris, Librairie du Recueil Sirey, 1942.

Cannat, P., Variétés, Revue Pénitentiaire et de droit pénal, 1949a, 1, pp. 40-44.

Cannat, P., La Réforme pénitentiaire, Cours enseigné au centre d'études de Fresnes, Paris, Librairie du Recueil Sirey, 1949b.

82 Alphonse Boudard a évoqué les ambiguittés des évaluations menées au Centre d'orientation de Fresnes dans le cadre de la réforme pénitentiaire (2000, pp. 406-432). Georges Arnaud avait fait de même lors de son reportage, Prisons 53. Arnaud (1953). 
Cannat, P., Chronique de criminologie, un type de relégué, Revue de Science criminelle et de droit pénal comparé, 1950a, 1, pp. 78-81.

Cannat, P., Les nouvelles tendances du droit pénal aux États-Unis, Revue de Science criminelle et de droit pénal comparé, 1950b, 2, pp. 157-170.

Cannat, $\mathrm{P}$., Chronique de criminologie, la déception facteur criminogène, Revue de Science criminelle et de droit pénal comparé, 1951, 4, pp. 549-552.

Cannat, P., La semi-liberté, Revue de Science criminelle et de droit pénal comparé, 3, 1953, pp. 328-332.

Cannat P., La rééducation des délinquants récidivistes, Revue pénitentiaire et de droit pénal, 1955, 4, pp. 514-594.

Collectif, Actes du Ilème Congrès international de criminologie, tome II, Paris, PUF, 1952.

Di Tullio, B., Manuel d'anthropologie criminelle, Paris, Payot, 1951.

Dublineau, J., L'antisocialité est-elle une catégorie ou une définition?, Revue de Science criminelle et de droit pénal comparé, 1949, 4, pp. 783-789.

Dupré, E., Pathologie de l'imagination et de l'émotivité, Paris, Payot, 1925.

Finelli, R., Inspecteur de l'administration, Étude de 282 relégués à Saint-Martin-de-Ré en 1949, Revue pénitentiaire et de droit pénal, 1951, 1, pp. 43-48.

Frank E. E., Observation, triage et rééducation, Revue de Science criminelle et de droit pénal comparé, 1939, 2, pp. 36-62.

Grapin, P., Esquisse d'une nouvelle perspective anthropo-criminologique, Revue de Science criminelle et de droit pénal comparé, 1949, 1, pp. 37-45.

Grapin, P., Dysmorphisme et criminalité, Revue de Science criminelle et de droit pénal comparé, 1954, 1, pp. 65-81.

Pella, V., Rapport sur les travaux de la Commission sociale de l'ONU, le 30 mars 1949 à Lake-Sucess sur les mesures préventives contre le crime et le traitement des délinquants, Revue pénitentiaire et de droit pénal, 1950, 1, p. 137.

Perdriau, A., Les développements récents dans le domaine de l'examen médico-psychologique et social des délinquants en France, Revue de Science criminelle et de droit pénal comparé, 1955, 2, pp. 277-305.

Perdriau, A., Réunion des présidents et assistantes sociale des Comités d'assistance aux libérés les 28-29 mai 1956, Revue pénitentiaire et de droit pénal, 1956, 4, pp. 887-897.

Pinatel, J., Les diverses conceptions de la science pénitentiaire, Revue de Science criminelle et de droit pénal comparé, 1949, 4, pp. 717-722.

Pinatel, J., Chronique de criminologie, l'examen médico-psychologique et social des délinquants, Revue de Science criminelle et de droit pénal comparé, 1953, 2, pp. 702-706.

Pinatel, J., Hérédité et criminalité, Revue de Science criminelle et de droit pénal comparé, 1954, 4, pp. 574-586.

Pinatel, J., La criminologie, Paris, Spes, 1960, réédition Éditions ouvrières, 1969.

Touren, A., Dix années de réforme pénitentiaire, Revue pénitentiaire et de droit pénal, 1956, 2, pp. 416-431.

Vimont, J.-C., L'œuvre salutiste de Radepont et l'assistance aux relégués, Mémoires de la protection sociale en Normandie, 2005, 4, pp. 103-124.

Vullien, R., Le problème des relégués, réflexions d'ordre psychiatrique, Revue pénitentiaire et de droit pénal, 1950, 1, pp. 105-112. 